\title{
Dexmedetomidine alleviates insulin resistance in hepatocytes by reducing endoplasmic reticulum stress
}

\author{
Fanfan $\mathrm{Liu}^{1} \cdot$ Shaojun $\mathrm{Zhu}^{1} \cdot{\text { Lifeng } \mathrm{Ni}^{1} \cdot \text { Ling'er Huang }^{1} \cdot \text { Kuirong Wang }}^{1} \cdot$ Yanfeng Zhou ${ }^{1}$
}

Received: 5 July 2019 / Accepted: 16 October 2019 / Published online: 2 November 2019

(C) The Author(s) 2019

\begin{abstract}
Purpose Dexmedetomidine (DEX) stabilizes intraoperative blood glucose levels and reduces insulin resistance (IR), a common perioperative complication. However, the molecular mechanisms underlying these effects remain unclear. Since endoplasmic reticulum stress (ERS) is a mechanism of IR, this study sought to examine whether DEX can effectively alleviate IR by reducing ERS.

Methods HepG2 and LO2 cells were treated with different concentrations of insulin. The glucose content assay and Cell Counting Kit-8 (CCK-8) were then employed to determine the optimal insulin concentration capable of inducing IR without affecting cell viability. Insulin-resistant hepatocytes were cultured with different concentrations of DEX for $24 \mathrm{~h}$, and the glucose concentration in the supernatant was measured. ERS was assessed by qPCR and western blotting. The latter was also used to quantify the expression of phosphorylated protein kinase B (p-AKT), phosphoenolpyruvate carboxykinase (PEPCK), and glucose 6 phosphatase (G6Pase), which are key proteins involved in the action of insulin.

Results After 48-h of culturing with $10 \mu \mathrm{g} / \mathrm{mL}$ insulin, glucose consumption in hepatocytes was found to be reduced. IR hepatocytes cultured with 10,100 , or $1000 \mathrm{ng} / \mathrm{ml}$ DEX for $24 \mathrm{~h}$ showed a concentration-dependent increase in glucose consumption. Elevated mRNA and protein levels of ERS markers binding immunoglobulin protein (BIP) and ER protein 29 (ERp29), were reversed by DEX treatment. Moreover, reduced p-AKT and increased PEPCK and G6Pase protein levels in IR hepatocytes were also restored following DEX treatment.

Conclusion DEX may alleviate IR in hepatocytes by reducing ERS serving to restore insulin action via the IRS-1/PI3K/AKT pathway.
\end{abstract}

Keywords Dexmedetomidine $\cdot$ Insulin resistance $\cdot$ Endoplasmic reticulum stress $\cdot$ Hepatocytes $\cdot$ AKT

\section{Introduction}

Insulin resistance (IR) results from impaired glucose metabolism, and is characterized by a decreased sensitivity of target organs to insulin. IR is one of the most common and serious perioperative complications and is frequently associated with a longer hospital stay, increased susceptibility to infection, and higher risk of mortality [1-3].

These authors contributed equally: Fanfan Liu, Shaojun Zhu

Yanfeng Zhou

zhouyf11@zju.edu.cn

1 Department of Anesthesiology, The First Affiliated Hospital, College of Medicine, Zhejiang University, Hangzhou, Zhejiang, PR China
The liver plays an important role in glucose metabolism, although adipose tissue and skeletal muscle are also targeted by insulin. IR in the liver can lead to increased gluconeogenesis and glycogen output, resulting in fasting hyperglycemia and hyperinsulinemia. On the other hand, fat mobilization and fatty acid oxidation are inhibited by insulin, and the consequent elevation in free fatty acid levels can act on insulin signaling pathways in hepatocytes to aggravate hepatic IR [4-6].

Endoplasmic reticulum stress (ERS) is an important mechanism of IR [7] involving protein kinase-like ER kinase (PERK), activating transcription factor (ATF) 6, and inositol-requiring enzyme (IRE) $1 \alpha$, which are maintained in an inactive state in the endoplasm by glucose regulated protein 78 (also known as binding immunoglobulin protein [BIP]) under normal conditions. Under conditions of hypoxia or excess sugar, the number of misfolded proteins increases; the above proteins dissociate from BIP and are 
activated, causing c-Jun N-terminal kinase-regulated insulin receptor substrate phosphorylation and in extreme cases, apoptosis through the CCAAT/enhancer-binding protein homologous protein pathway [8]. The insulin receptor substrate-1/phosphatidylinositol 3-kinase/protein kinase B (IRS-1/PI3K/AKT) pathway is a classical pathway for insulin action. Tyrosine phosphorylation of IRS-1 and binding to PI3K promote AKT activation, mainly at Thr308 and Ser473 sites [9]. It has been confirmed that ERS eventually inhibits the IRS-1/PI3K/AKT signaling pathway to inhibit insulin action in liver cells [8]. PEPCK and G6Pase, downstream effectors of the IRS-1/PI3K/AKT pathway, are key enzymes for gluconeogenesis and glycogenolysis respectively, and are directly related to changes in blood glucose [10].

Dexmedetomidine (DEX), a novel $\alpha 2$ adrenergic agonist with sedative, analgesic, anti-inflammatory, and organprotective effects, is widely used in anesthesia and intensive care [11-13]. DEX can maintain blood glucose stability and reduce blood glucose levels, which may be associated with the suppression of systemic inflammation and pain and the regulation of humoral immunity and complement function [13]. In burn and ischemia-reperfusion models, DEX has been shown to protect organ function by reducing ERS levels $[14,15]$. Based on these findings, we speculated that DEX can stabilize blood glucose and relieve IR by reducing ERS and promoting the conduction of the IRS-1/PI3K/AKT pathway in the liver.

\section{Materials and methods}

\section{Cell lines and culture}

Human HepG2 and LO2 hepatoma cell lines were provided by the Research Group of Hepatobiliary Surgery, Key Laboratory of Organ Transplantation of Zhejiang Provence, China. The cells were grown in minimal Eagle's medium (MEM; Gino Bio, Hangzhou, China) and Dulbecco's modified Eagle's medium (DMEM; Gino Bio, Hangzhou, China), respectively, supplemented with $10 \%$ fetal bovine serum (Wisent, SaintJean-Baptiste, QC, Canada) and $1 \%$ penicillin-streptomycin in a $5 \% \mathrm{CO}_{2}$ atmosphere at $37^{\circ} \mathrm{C}$. Cells were used for experiments when they reached $90 \%$ confluence.

\section{Cell viability assay}

Cell viability was evaluated with a Cell Counting Kit (CCK)-8 (Dojindo Laboratories, Kumamoto, Japan). Approximately $5 \times 10^{3}$ HepG 2 cells and $1 \times 10^{4}$ LO2 cells were seeded in each well of a 96-well plate. Medium containing $10 \%$ CCK-8 reagent was added to the well, followed by incubation for $1-3 \mathrm{~h}$ at $37^{\circ} \mathrm{C}$. When the color of the medium turned dark orange, optical density at $450 \mathrm{~nm}$ $\left(\mathrm{OD}_{450}\right)$ was measured and a standard curve of cell viability was generated [16].

\section{Analysis of glucose content}

The glucose content of the medium was measured by the glucose oxidase-peroxidase method using a commercial kit (Rsbio, Shanghai, China). HepG2 and LO2 cells were seeded as described above and cultured in the presence of insulin (Sigma-Aldrich, St. Louis, MO, USA) or DEX (Jiangsu Hengrui Medicine Co., Lianyungang, China). The medium was changed to serum- and Phenol Red-free DMEM or MEM (Wisent) for $12 \mathrm{~h}$, and the mixture of R1 and $\mathrm{R} 2$ in the kit was added to the supernatant at a ratio of 1:200 according to the instructions, followed by incubation at $37^{\circ} \mathrm{C}$ for $15 \mathrm{~min}$. $\mathrm{OD}_{505}$ was measured and a standard curve of glucose content was generated. Glucose consumption was calculated as the difference in the glucose contents of experimental and blank wells [17, 18].

\section{Model of IR}

Approximately $5 \times 10^{3}$ HepG2 cells and $1 \times 10^{4}$ LO2 cells were seeded in each well of a 96-well plate. When the cells were attached, medium containing different concentrations of insulin $(0,1,2.5,5,10$, and $20 \mu \mathrm{g} / \mathrm{mL})$ was added and the cells were cultured for 24,48 , and $72 \mathrm{~h}[17,19,20]$. The insulin concentration that caused the largest decrease in glucose consumption without affecting cell viability was selected as the IR model.

\section{Quantitative real-time (qRT-) PCR}

BIP and ER protein (ERp)29 mRNA levels were evaluated by qRT-PCR. Cells were washed with phosphate-buffered saline (PBS) and the total RNA was extracted using TRIzol reagent (Solar Bio, Beijing, China); cDNA was synthesized with the PrimeScript RT Reagent Kit with gDNA Eraser (Takara Bio, Otsu, Japan), and qRT-PCR was performed using SYBR Premix Ex Taq II (Tli RNaseH Plus; Takara Bio) under the following conditions: $95^{\circ} \mathrm{C}$ for $30 \mathrm{~s}$, followed by 40 cycles of $95^{\circ} \mathrm{C}$ for $5 \mathrm{~s}$ and $60^{\circ} \mathrm{C}$ for $30 \mathrm{~s}$. The following forward and reverse primers were used: ERp29, 5'-CCTGGATACGGTCACTTTCTACA-3' and 5'-AGTTT TCAGCAAGACGCTTGA-3'; BIP, 5'-GAGATCATCG CCAACGATCAG-3' and 5'-ACTTGATGTCCTGCTGC ACAG-3'; and glyceraldehyde 3-phosphate dehydrogenase (control), 5'-ACTTTGGTATCGTGGAAGGACTCAT-3' and R:5'-GTTTTTCTAGACGGCAGGTCAGG-3'. The relative expression levels of BIP and ERp29 were determined with the comparative cycle threshold $\left(2^{-\Delta \Delta C t}\right)$ method. 


\section{Western blot analysis}

After two washes with PBS, cells were lysed with buffer containing $2 \%$ phenylmethylsulfonyl fluoride and $1 \%$ protease inhibitor cocktail. Protein concentration was measured with a bicinchoninic acid assay kit. Equal amounts of protein $(\sim 30 \mu \mathrm{g})$ were separated by $10 \%$ sodium dodecyl sulfate-polyacrylamide gel electrophoresis and transferred to a polyvinylidene difluoride membrane (Merck Millipore, Billerica, MA, USA) for $\sim 100$ min. After blocking with $5 \%$ milk, the membrane was incubated overnight at $4{ }^{\circ} \mathrm{C}$ in a $1.25 \%$ milk solution containing primary antibodies including anti-BIP, antiAKT and anti-pAKT, (1:1000; Cell Signaling Technology, Danvers, MA, USA; Cat: \#3183; \#4060; \#4691), ERp29 (1:2000) and G6Pase, (1:500; Abcam, Cambridge, MA, USA; Cat:ab176573; ab83690), PEPCK, (1:2000; Proteintech Group, Chicago, IL, USA; Cat:16754-1-AP). The membrane was washed thrice with Tris-buffered saline containing $0.1 \%$ Tween-20, and incubated for $2 \mathrm{~h}$ at room temperature with a $1.25 \%$ milk solution containing horseradish peroxidase-conjugated goat anti-rabbit $\mathrm{IgG}$ (1:2000; Beyotime Institute of Biotechnology, Shanghai, China; Cat:A0208). Protein bands were developed by enhanced chemiluminescence (Fude Bio, Hangzhou, China) and signal intensity was determined using Image Lab software (Bio-Rad, Hercules, CA, USA).

\section{Statistical analysis}

Data were obtained from at least three independent experiments and are reported as the mean \pm SD. Statistical analyses were performed using SPSS v.13.0 (Chicago, IL, USA). Analysis of variance was used for multiple group comparisons. Statistical significance was set at $P<0.05$.

\section{Results}

\section{Establishment of the hepatic IR model}

In order to establish a model of hepatic IR, HepG2, and LO2 cells were incubated in a medium containing different concentrations of insulin $(0,1,2.5,5,10$, and $20 \mu \mathrm{g} / \mathrm{mL}$ ) for 24,48 , and $72 \mathrm{~h}$. Treatment with $20 \mu \mathrm{g} / \mathrm{ml}$ insulin for $24 \mathrm{~h}$ markedly reduced cell viability compared with the blank control group $(P<0.05$; Figs 1 and 2$)$. On the other hand, treatment with $10 \mu \mathrm{g} / \mathrm{mL}$ insulin for $72 \mathrm{~h}$ reduced cellular activity relative to the control cells $(P<$ $0.05)$, as evidenced by the decrease in glucose consumption, but had no effect on cell viability within the first $48 \mathrm{~h}$. The greatest decreases in glucose consumption were observed with $10 \mu \mathrm{g} / \mathrm{mL}$ insulin treatment for $48 \mathrm{~h}$ and $5 \mu \mathrm{g} / \mathrm{mL}$ insulin treatment for $72 \mathrm{~h}(P<0.05)$, indicating that cells in these groups showed the strongest IR.
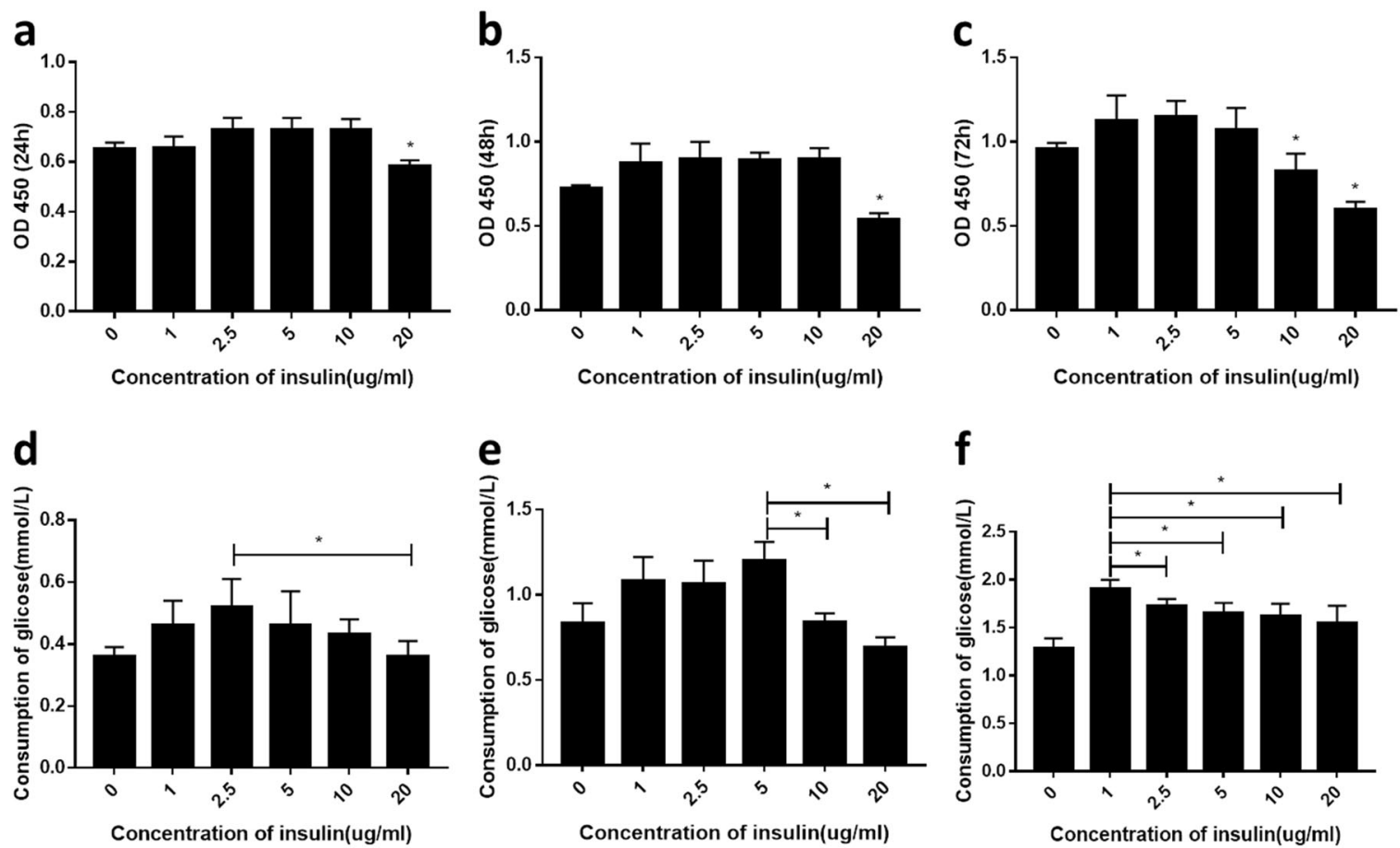

Fig. 1 Media containing different concentrations of insulin affect viability and glucose consumption in HepG 2 cells $(\mathbf{a}-\mathbf{f})$, cell viability (a-c), and glucose consumption $(\mathbf{d}-\mathbf{f})$ at $24 \mathrm{~h}(\mathbf{a}, \mathbf{d}), 48 \mathrm{~h}(\mathbf{b}, \mathbf{e})$, and
$72 \mathrm{~h}(\mathbf{c}, \mathbf{f})$. A representative histogram from three individual experiments is shown, and data are presented as mean \pm SD. $* P<0.05$ vs. blank group 

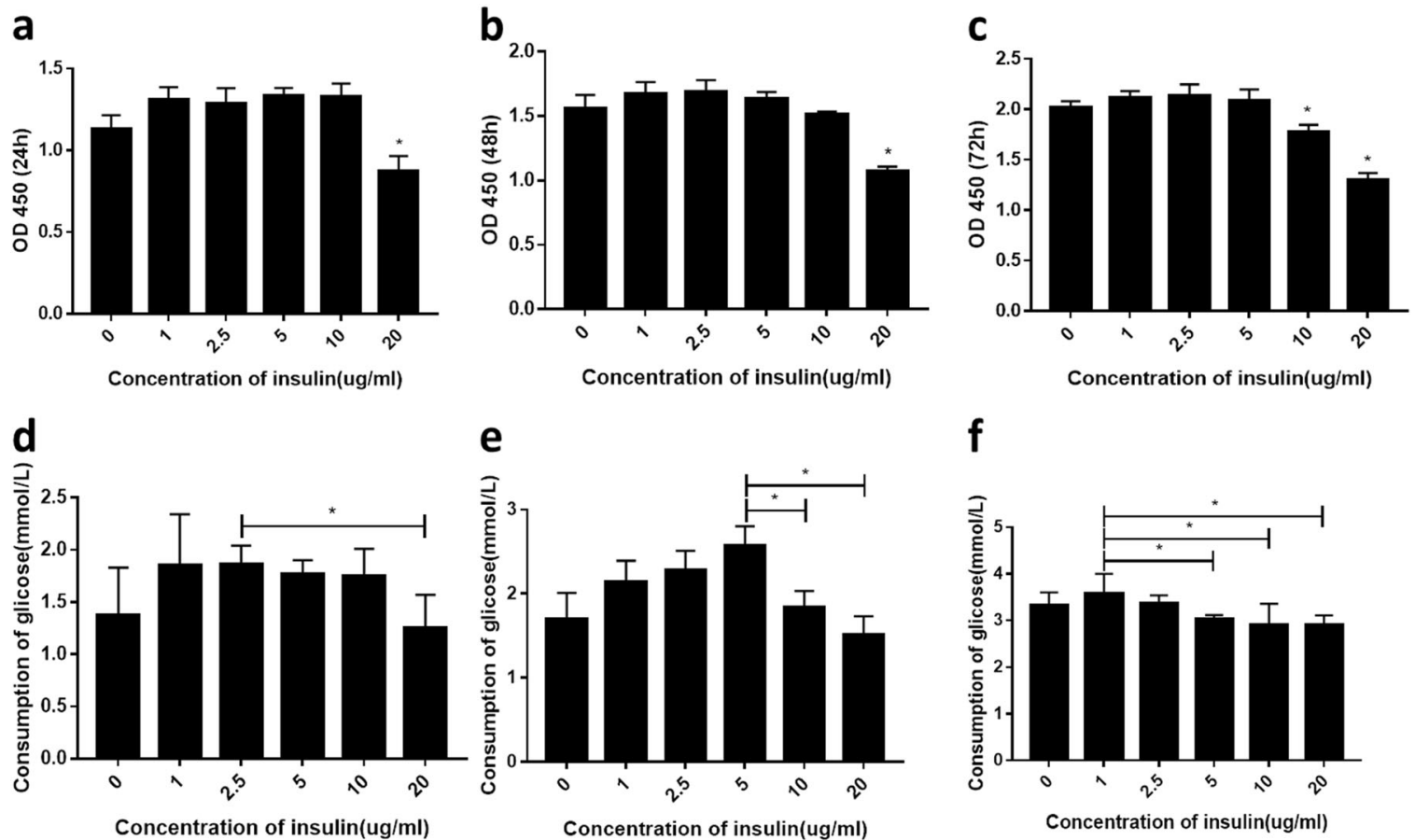

Fig. 2 Media containing different concentrations of insulin affect viability and glucose consumption in LO2 cells (a-f), cell viability (a-c), and glucose consumption (d-f) at $24 \mathrm{~h}(\mathbf{a}, \mathbf{d}), 48 \mathrm{~h}(\mathbf{b}, \mathbf{e})$, and

$72 \mathrm{~h}(\mathbf{c}, \mathbf{f})$. A representative histogram from three individual experiments is shown, and data are presented as mean \pm SD. $* P<0.05$ vs. blank group
Table 1 Effects of DEX concentration on the viability of HepG2 and LO2 cells

\begin{tabular}{|c|c|c|c|c|c|}
\hline \multicolumn{2}{|c|}{$\begin{array}{l}\text { Concentration of } \\
\text { DEX }(\mathrm{ng} / \mathrm{ml})\end{array}$} & 0 & 10 & 100 & 1000 \\
\hline $\mathrm{OD}_{450}$ & HepG2 & $0.795 \pm 0.041$ & $0.849 \pm 0.027$ & $0.837 \pm 0.094$ & $0.825 \pm 0.046$ \\
\hline & LO2 & $1.317 \pm 0.044$ & $1.287 \pm 0.062$ & $1.315 \pm 0.067$ & $1.310 \pm 0.115$ \\
\hline
\end{tabular}

Data are shown as mean \pm SD. Viability did not differ relative to the blank group
However, cells cultured in the presence of insulin for $72 \mathrm{~h}$ appeared unhealthy and the culture medium was cloudy. We therefore used $10 \mu \mathrm{g} / \mathrm{mL}$ insulin treatment for $48 \mathrm{~h}$ as the conditions to generate the IR model.

\section{DEX has no effect on HepG2 and LO2 cell viability}

To exclude the effect of reduced cell viability on glucose consumption, we cultured HepG2 and LO2 cells with different concentrations of $\operatorname{DEX}(10,100$, and $1000 \mathrm{ng} / \mathrm{mL})$ $[21,22]$ and evaluated cell viability. There were no differences in viability between DEX-treated and control cells $(P>0.05$; Table 1), indicating that DEX is not toxic to hepatocytes.

\section{DEX alleviates IR in a concentration-dependent manner}

We tested the stability of the hepatic IR model and found no difference in glucose consumption in the first $24 \mathrm{~h}$ relative to the IR group. However, when cells were cultured in the medium containing DEX, glucose consumption was increased compared with that in the 24 -h IR group $(P<$ 0.05; Table 2). We also observed that glucose consumption increased with DEX concentration, suggesting that DEX alleviates IR in a concentration-dependent manner.

\section{DEX alleviates IR by inhibiting BIP and ERp29 expression}

BIP and ERp29 are markers of ERS; the mRNA and protein levels of the two markers were higher in the IR group than in the control group $(P<0.05$; Fig. 3$)$, suggesting that IR is closely related to ERS. However, both markers were downregulated in the DEX group compared to that in the IR group $(P<0.05)$, indicating that DEX reduced insulinassociated ERS in hepatocytes.

\section{DEX restores the reduced phosphorylation of AKT}

To investigate whether DEX restores insulin action by reducing ERS, AKT, and p-AKT were detected by western 
Table 2 Effects of DEX concentration on glucose consumption and stability of hepatocytes

\begin{tabular}{|c|c|c|c|c|c|c|}
\hline \multirow[t]{2}{*}{ Group } & & \multirow[t]{2}{*}{ IR } & \multirow[t]{2}{*}{ IR-24 h } & \multicolumn{3}{|c|}{ Concentration of DEX $(\mathrm{ng} / \mathrm{ml})$} \\
\hline & & & & 10 & 100 & 1000 \\
\hline \multirow{2}{*}{$\begin{array}{l}\text { Consumption of glucose } \\
(\mathrm{mmol} / \mathrm{L})\end{array}$} & HepG2 & $0.91 \pm 0.06$ & $0.93 \pm 0.16$ & $1.10 \pm 0.02 *$ & $1.29 \pm 0.13^{*}$ & $1.32 \pm 0.13 *$ \\
\hline & LO2 & $2.79 \pm 0.31$ & $2.76 \pm 0.20$ & $3.52 \pm 0.15^{*}$ & $3.65 \pm 0.17 *$ & $3.98 \pm 0.18 *$ \\
\hline
\end{tabular}

Data are shown as mean \pm SD. $* P<0.05$ vs. IR group

a

Fig. 3 DEX reduces the expression of ERS proteins BIP and ERp29 in HepG2 and LO2 cells $(\mathbf{a}-\mathbf{f})$. The levels of protein (a-d) and mRNA $(\mathbf{e}, \mathbf{f})$ in HepG2 cells (a, c and e) and LO2 cells (b, $\mathbf{d}$ and $\mathbf{f})$. A representative histogram from three individual experiments is shown, and data are presented as mean \pm SD. ${ }^{\#} P<0.05$ vs. normal $(\mathrm{N})$ group; $* P<0.05$ vs. IR group

C
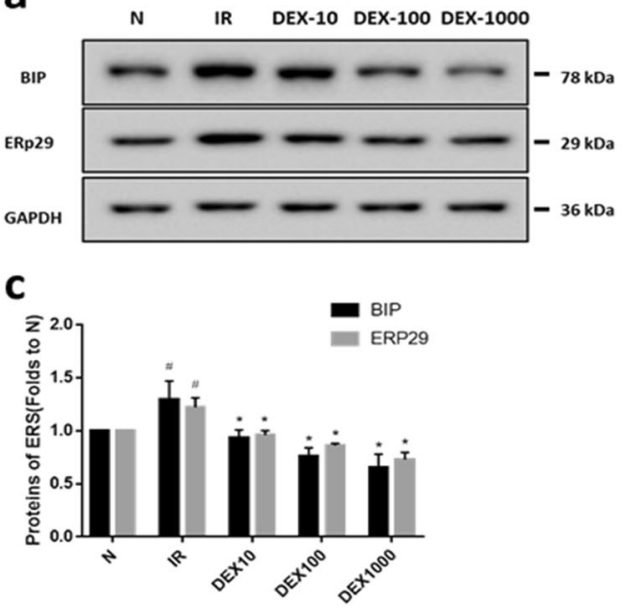

e

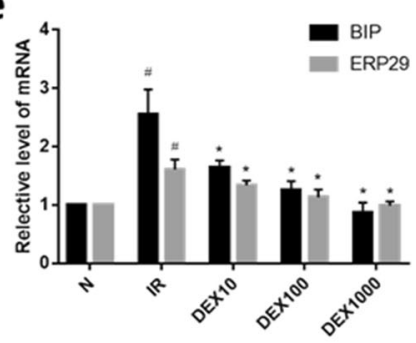

b

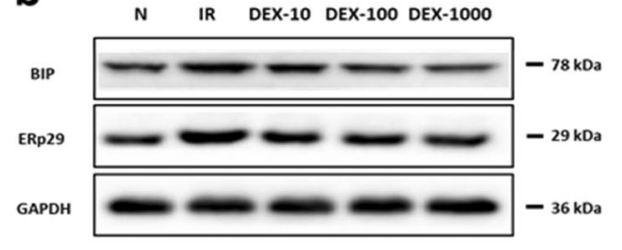

d

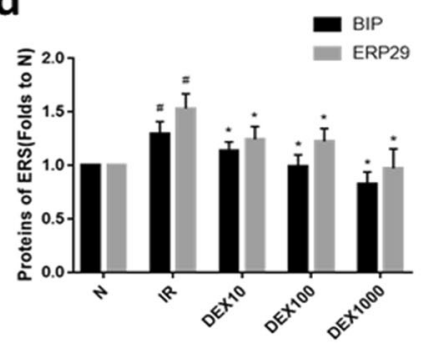

f

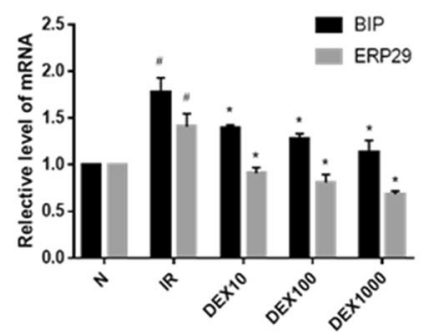

blot. High concentrations of insulin significantly decreased insulin-stimulated phosphorylation of AKT at serine 473 $(P<0.05$; Fig. 4$)$, which is an important event in the insulin signaling pathway. DEX treatment restored this change and significantly increased serine phosphorylation of AKT $(P<$ 0.05; Fig. 4). Hence, it is suggested that DEX may restore insulin action via the IRS-1/PI3K/AKT pathway.

\section{DEX restores increased gluconeogenesis and glycogenolysis}

Gluconeogenesis and glycogenolysis are important mechanisms associated with glucose metabolism in the liver; with PEPCK and G6Pase acting as key enzymes within these pathways, respectively. The expression of PEPCK and G6Pase proteins was significantly increased $(P<0.05$; Fig. 5) in high insulin-induced IR hepatocytes, however, they were found to be significantly decreased
$(P<0.05$; Fig. 5) following treatment with different concentrations of DEX. This indicates that DEX may eventually reduce gluconeogenesis and glycogenolysis in the liver, thereby serving to maintain blood glucose stability.

\section{Discussion}

In this study, we showed that IR is associated with ERS. Treatment of IR hepatocytes with DEX increased glucose consumption, implying that DEX can alleviate IR; this was accompanied by downregulation of BIP and ERp29. These results indicate that DEX alleviates hepatic IR by decreasing ERS via suppression of the ERS proteins BIP and ERp29. Meanwhile, the increase in p-AKT and reduction in PEPCK and G6Pase also indicate that DEX may restore insulin activity through the IRS-1/PI3K/AKT pathway while inhibiting gluconeogenesis and glycogenolysis. 
Fig. 4 DEX restores the reduced phosphorylation of AKT in HepG2 and LO2 cells (a-d). The relative levels of protein of pAKT/AKT in HepG2 cells (a, c) and $\mathrm{LO} 2$ cells $(\mathbf{b}, \mathbf{d})$. A representative histogram from three individual experiments is shown, and data are presented as mean \pm SD. ${ }^{\#} P<0.05$ vs. normal $(\mathrm{N})$ group; $* P<0.05$ vs. IR group

Fig. 5 DEX restores the increased PEPCK and G6Pase in HepG2 and LO2 cells (a-d) The relative expression levels of PEPCK and G6Pase proteins in HepG2 cells $(\mathbf{a}, \mathbf{c})$ and $\mathrm{LO} 2$ cells $(\mathbf{b}, \mathbf{d})$. A representative histogram is from three individual experiments. Data are presented as mean \pm SD.

${ }^{\#} P<0.05$ vs. normal $(\mathrm{N})$ group; $* P<0.05$ vs. IR group a

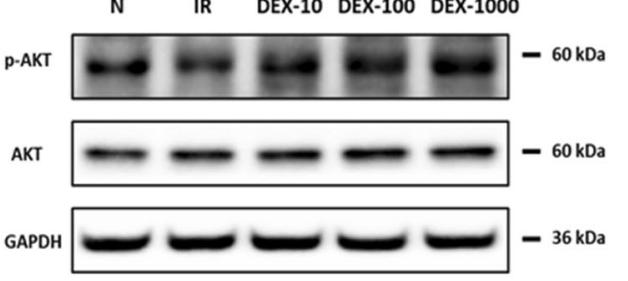

c

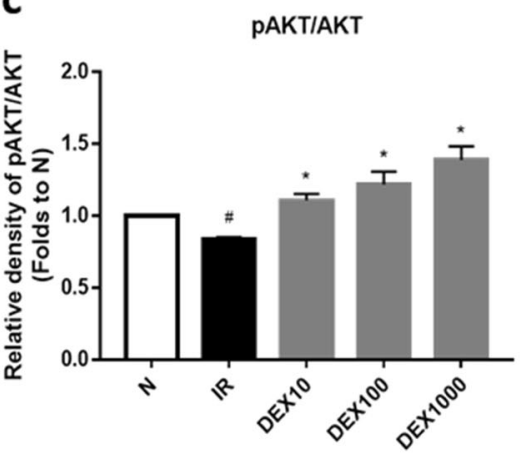

a
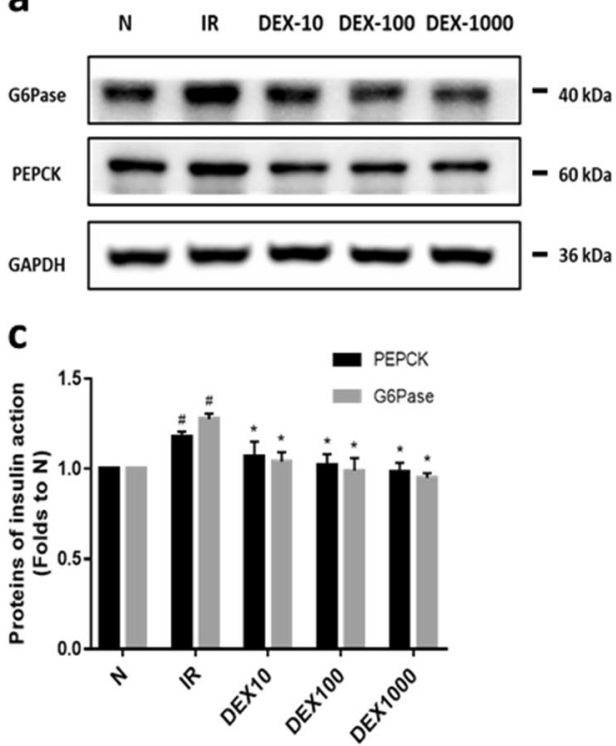

b

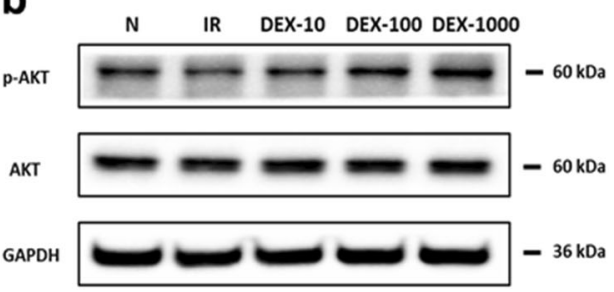

d

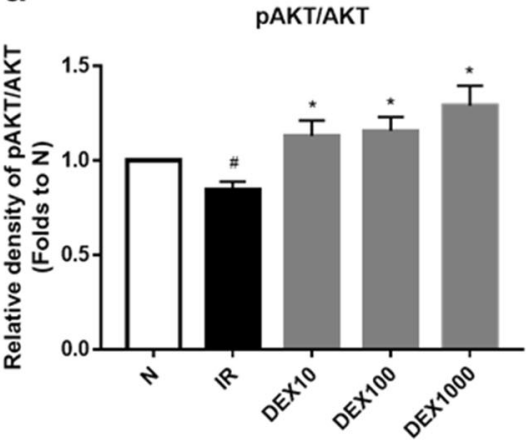

b
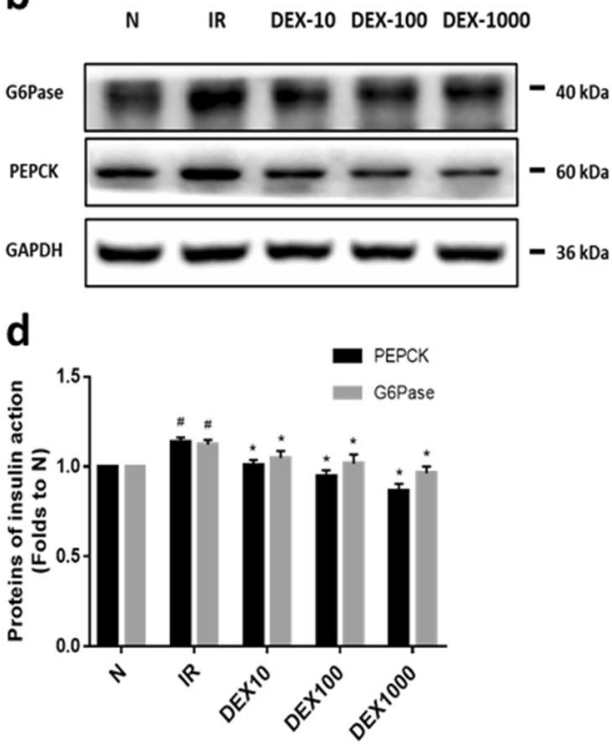

HepG2 cells are derived from human hepatocarcinoma tissue, which secretes a variety of plasma proteins, including albumin, $\alpha 2$-macroglobulin, plasminogen, and iron transfer protein; these cells are similar to normal human hepatocytes in terms of their biochemical characteristics and biosynthetic capacities [23]. The liver synthesizes, stores, and releases glycogen, which is important for glucose metabolism. Therefore, HepG2 cells are an ideal cell model for investigating the mechanisms of IR and testing the efficacy of hypoglycemic drugs $[24,25]$. We also used LO2 cells, which are normal hepatocytes, for further validation in this study.
In vitro IR models are mainly generated in hepatocytes, fat cells, and skeletal muscle cells using high concentrations of insulin; medium containing high levels of sugar and fat; dexamethasone; palmitic acid; and tunicamycin [26-29], although a high insulin concentration is most frequently used [30]. Dexamethasone, a kind of glucocorticoid, is easily confused with dexmedetomidine (owing to similar abbreviations), which induces IR by inhibiting the binding of insulin to the insulin receptor. In this study, we determined that an insulin concentration of $10 \mu \mathrm{g} / \mathrm{ml}$ applied to HepG2 and LO2 cells for $48 \mathrm{~h}$ resulted in the lowest glucose consumption while cell viability was unaffected. In 
previous studies, glucose consumption in normal cells was used as the control $[24,25]$, but we found that insulin has a mitogenic effect on hepatocytes, although there were no changes in proliferation rate within a certain concentration range. This has also been described by other investigators $[31,32]$. Since cell proliferation affects glucose consumption, we compared cells with similar proliferation rates but selected those with the highest glucose consumption within each group as the control; IR was identified based on a decrease in glucose consumption. The group showing the largest difference in glucose consumption was considered the optimal model. We believe that this method is more objective than the approaches used in previous studies.

The three major ERS proteins are PERK, ATF6, and IRE1 $\alpha$; these are normally bound by BIP and maintained in an inactive state, and dissociate from BIP upon ERS to activate downstream effectors. Thus, intracellular BIP level can serve as a marker of ERS [6, 8]. ERp29 is a soluble protein located on the endoplasmic reticulum membrane [33]; ERp29 mRNA and protein levels were found to increase with BIP expression in a tunicamycin-induced ERS model of Ins-1 islet cells; additionally, ERp29 was shown to be involved in ER stress-mediated islet cell damage [34]. The occurrence of ERS further inhibits insulin action through the IRS-1/PI3K/AKT pathway as one of the downstream mechanisms of ERS [8]. Phosphorylation of IRS- 1 at serine sites and decreased phosphorylation of AKT result in disrupted insulin signaling and induction of insulin resistance. Liver-specific gluconeogenesis and glycogenolysis have a direct impact on the regulation of blood glucose, with PEPCK and G6Pase serving as particularly important factors in these two pathways [10].

Clinical studies have demonstrated that DEX stabilizes perioperative stress-induced hyperglycemia and decreases IR in patients with diabetes; this may be related to a reduction in sympathetic activity, analgesia, and antiinflammatory response [13]. DEX exerted protective effects in lungs by reducing the levels of ERS in a model of ischemia-reperfusion injury [35], and was shown to be closely related to ERS caused by burns and glucose deprivation $[12,14,16]$. However, the effects of DEX on ERS in an IR model have not been previously reported. Our results demonstrate that DEX can stabilize blood glucose level by reducing ERS and restoring insulin action. It is known that ERS is associated with the release of inflammatory factors, which may indirectly affect nuclear factor- $\mathrm{kB}$ signaling [35]. Whether DEX further reduced ERS by suppressing inflammation to affect blood glucose level is unclear, since we only measured glucose consumption in the medium. Furthermore, although we observed that DEX inhibited the expression of ERS proteins, the role of ERS in IR was not confirmed by gene knockout experiments. Additional studies are also needed to clarify the role of inflammation in this process. The IRS-1/PI3K/AKT pathway in the liver is inhibited during IR. The phosphorylation of AKT is reduced, resulting in the activation of glycogen synthase kinase-3 (GSK-3), which triggers glycogen synthase (GS) phosphorylation and interferes with glycogen synthesis [36]. Although we did not directly examine the effects of DEX on GSK-3 and GS, we did observe that DEX reduced the levels of gluconeogenesis and glycogenolysis in the liver. Therefore, additional in vivo experiments are required to provide further evidence for clinical work. Nonetheless, our findings indicate that treatment with DEX may be an effective intervention for preventing perioperative IR and provide novel insights into the molecular basis for its beneficial effects.

Funding This study was funded by grants from the Department of Science and Technology of Zhejiang province (391010-I50904).

\section{Compliance with ethical standards}

Conflict of interest The authors declare that they have no conflict of interest.

Ethical approval This article does not contain any studies with human participants or animals performed by any of the authors.

Publisher's note Springer Nature remains neutral with regard to jurisdictional claims in published maps and institutional affiliations.

Open Access This article is distributed under the terms of the Creative Commons Attribution 4.0 International License (http://crea tivecommons.org/licenses/by/4.0/), which permits unrestricted use, distribution, and reproduction in any medium, provided you give appropriate credit to the original author(s) and the source, provide a link to the Creative Commons license, and indicate if changes were made.

\section{References}

1. S. Behdad, A. Mortazavizadeh, V. Ayatollahi, Z. Khadiv, S. Khalilzadeh, The effects of propofol and isoflurane on blood glucose during abdominal hysterectomy in diabetic patients. Diabetes Metab. J. 38, 311-316 (2014)

2. K. Højlund, Metabolism and insulin signaling in common metabolic disorders and inherited insulin resistance. Dan. Med J. 61, B4890 (2014)

3. L. Zhou, L. Wang, B. Yang, J. Zeng, Q. Zhang, H. Lei, S. Xu, Protective effect of pretreatment with propofol against tumor necrosis factor- $\alpha$-induced hepatic insulin resistance. Exp. Ther. Med. 10, 289-294 (2015)

4. P. Staehr, O. Hother-Nielsen, H. Beck-Nielsen, The role of the liver in type 2 diabetes. Rev. Endocr. Metab. Disord. 5, 105-110 (2004)

5. R. Basu, V. Chandramouli, B. Dicke, B. Landau, R. Rizza, Obesity and type 2 diabetes impair insulin-induced suppression of glycogenolysis as well as gluconeogenesis. Diabetes 54, 1942-1948 (2005)

6. A.L. Birkenfeld, G.I. Shulman, Nonalcoholic fatty liver disease, hepatic insulin resistance, and type 2 diabetes. Hepatology 59, 713-723 (2014) 
7. U. Ozcan, Q. Cao, E. Yilmaz, A.H. Lee, N.N. Iwakoshi, E. Ozdelen, G. Tuncman, C. Görgün, L.H. Glimcher, G.S. Hotamisligil, Endoplasmic reticulum stress links obesity, insulin action, and type 2 diabetes. Science 306, 457-461 (2004)

8. A. Chakrabarti, A.W. Chen, J.D. Varner, A review of the mammalian unfolded protein response. Biotechnol. Bioeng. 108, 2777-2793 (2011)

9. W. Chen, J. Wang, Y. Luo, T. Wang, X. Li, A. Li, J. Li, K. Liu, B. Liu, Ginsenoside Rb1 and compound $\mathrm{K}$ improve insulin signaling and inhibit ER stress-associated NLRP3 inflammasome activation in adipose tissue. J. Ginseng Res. 40, 351-358 (2016)

10. T.Y. Liu, C.X. Shi, R. Gao, H.J. Sun, X.Q. Xiong, L. Ding, Q. Chen, Y.H. Li, J.J. Wang, Y.M. Kang, G.Q. Zhu, Irisin inhibits hepatic gluconeogenesis and increases glycogen synthesis via the PI3K/Akt pathway in type 2 diabetic mice and hepatocytes. Clin. Sci. 129, 839-850 (2015)

11. R.D. Sanders, P. Sun, S. Patel, M. Li, M. Maze, D. Ma, Dexmedetomidine provides cortical neuroprotection: impact on anaesthetic-induced neuroapoptosis in the rat developing brain. Acta Anaesthesiol. Scand. 54, 710-716 (2010)

12. M. Ibacache, G. Sanchez, Z. Pedrozo, F. Galvez, C. Humeres, G. Echevarria, J. Duaso, M. Hassi, L. Garcia, G. Díaz-Araya, S. Lavandero, Dexmedetomidine preconditioning activates prosurvival kinases and attenuates regional ischemia/reperfusion injury in rat heart. Biochim. Biophys. Acta 1822, 537-545 (2012)

13. Y.S. Hui, C.Y. Suk, The effects of dexmedetomidine administration on postoperative blood glucose levels in diabetes mellitus patients undergoing spinal anesthesia: a pilot study. Anesth. Pain. Med. 6, e40483 (2016)

14. H. Wang, S. Zhang, S. Xu, L. Zhang, The efficacy and mechanism of dexmedetomidine in myocardial apoptosis via the reninangiotensin-aldosterone system. J. Renin Angiotensin Aldosterone Syst. 16, 1274-1280 (2015)

15. C. Liu, Q. Fu, R. Mu, F. Wang, C. Zhou, L. Zhang, B. Yu, Y. Zhang, T. Fang, F. Tian, Dexmedetomidine alleviates cerebral ischemia-reperfusion injury by inhibiting endoplasmic reticulum stress dependent apoptosis through the PERK-CHOP-Caspase-11 pathway. Brain Res. 1701, 246-254 (2018)

16. K. Wang, Y. Zhu, Dexmedetomidine protects against oxygenglucose deprivation/reoxygenation injury-induced apoptosis via the p38 MAPK/ERK signalling pathway. J. Int. Med. Res. 46, 675-686 (2018)

17. N. Qin, C.B. Li, M.N. Jin, L.H. Shi, H.Q. Duan, W.Y. Niu, Synthesis and biological activity of novel tiliroside derivants. Eur. J. Med. Chem. 46, 5189-5195 (2011)

18. R. Visvanathan, C. Jayathilake, R. Liyanage, A simple microplatebased method for the determination of $\alpha$-amylase activity using the glucose assay kit (GOD method). Food Chem. 211, 853-859 (2016)

19. F. Fang, M.L. Luo, N. Su, X.R. Wu, Effect of mulberry leaves extracts on glucose uptake of insulin-resistant HepG2 cells and the mechanism. Yao Xue Xue Bao 47, 1452-1456 (2012)

20. P. Lu, X. Chen, Z. Zhang, J. Zhang, Y. Yang, Z. Liu, J. Xie, S. Shao, X. Zhou, S. Hu, W. He, J. Zhao, X. Yu, Insulin upregulates betatrophin expression via PI3K/Akt pathway. Sci. Rep. 7, 5594 (2017)

21. Y. Zhang, X. Tan, L. Xue, The alpha2-adrenoreceptor agonist dexmedetomidine protects against lipopolysaccharide-induced apoptosis via inhibition of gap junctions in lung fibroblasts. Biochem. Biophys. Res. Commun. 495, 92-97 (2018)

22. J. Chen, Z. Jiang, X. Zhou, X. Sun, J. Cao, Y. Liu, X. Wang, Dexmedetomidine preconditioning protects cardiomyocytes against hypoxia/reoxygenation-induced necroptosis by inhibiting hmgb1-mediated inflammation. Cardiovasc Drugs Ther. 33, 45-54 (2019)

23. J.M. Choi, S.J. Oh, S.Y. Lee, J.H. Im, J.M. Oh, C.S. Ryu, H.C. Kwak, J.Y. Lee, K.W. Kang, S.K. Kim, HepG2 cells as an in vitro model for evaluation of cytochrome $\mathrm{P} 450$ induction by xenobiotics. Arch. Pharm. Res. 38, 691-704 (2015)

24. L. Zhao, X. Guo, O. Wang, H. Zhang, Y. Wang, F. Zhou, J. Liu, B. Ji, Fructose and glucose combined with free fatty acids induce metabolic disorders in HepG2 cell: a new model to study the impacts of high-fructose/sucrose and high-fat diets in vitro. Mol. Nutr. Food Res. 60, 909-921 (2016)

25. J. Nie, Y. Chang, Y. Li, Y. Zhou, J. Qin, Z. Sun, H. Li, Caffeic acid phenethyl ester (propolis extract) ameliorates insulin resistance by inhibiting JNK and NF- $\mathrm{KB}$ inflammatory pathways in diabetic mice and HepG2 cell models. J. Agric. Food Chem. 65, 9041-9053 (2017)

26. X.D. Wan, W.B. Yang, Y.Z. Xia, J.F. Wang, T. Lu, X.M. Wang, Disruption of glucose homeostasis and induction of insulin resistance by elevated free fatty acids in human L02 hepatocytes. J. Endocrinol. Investig. 32, 454-459 (2009)

27. L. Lei, Y. Zhu, W. Gao, X. Du, M. Zhang, Z. Peng, S. Fu, X. Li, W. Zhe, X. Li, G. Liu, Alpha-lipoic acid attenuates endoplasmic reticulum stress-induced insulin resistance by improving mitochondrial function in HepG2 cells. Cell. Signal. 28, 1441-1450 (2016)

28. L. Chen, H. Teng, H. Cao, Chlorogenic acid and caffeic acid from Sonchus oleraceus Linn synergistically attenuate insulin resistance and modulate glucose uptake in HepG2 cells. Food Chem. Toxicol. 127, 182-187 (2019)

29. Q. Zhang, X. Kong, H. Yuan, H. Guan, Y. Li, Y. Niu, Mangiferin improved palmitate-induced-insulin resistance by promoting free fatty acid metabolism in HepG2 and $\mathrm{C} 2 \mathrm{C} 12$ cells via PPAR $\alpha$ : mangiferin improved insulin resistance. J. Diabetes Res. 2019, 2052675 (2019)

30. P.H. Lou, E. Lucchinetti, L. Zhang, A. Affolter, M. Gandhi, A. Zhakupova, M. Hersberger, T. Hornemann, A.S. Clanachan, M. Zaugg, Propofol (Diprivan ${ }^{\circledR}$ ) and Intralipid $^{\circledR}$ exacerbate insulin resistance in type-2 diabetic hearts by impairing GLUT4 trafficking. Anesth. Analg. 120, 329-340 (2015)

31. D. Aizen, R. Sarfstein, I. Bruchim, D. Weinstein, Z. Laron, H. Werner, Proliferative and signaling activities of insulin analogues in endometrial cancer cells. Mol. Cell. Endocrinol. 406, 27-39 (2015)

32. C. Gu, P. Li, W. Liu, Y. Zhou, W.S. Tan, The role of insulin in transdifferentiated hepatocyte proliferation and function in serumfree medium. J. Cell. Mol. Med. 23, 4165-4178 (2019)

33. J. Wu, Y. Yang, S. Gao, H. Jiang, X.Q. Wang, Y. Xiao, X.H. Chen, P. Li, C.D. Xu, ERp29 inhibits tumorigenicity by suppressing epithelial mesenchymal transition in gastric cancer. Oncotarget 8, 78757-78766 (2017)

34. J. Gao, Y. Zhang, L. Wang, L. Xia, M. Lu, B. Zhang, Y. Chen, L. $\mathrm{He}$, Endoplasmic reticulum protein 29 is involved in endoplasmic reticulum stress in islet beta cells. Mol. Med. Rep. 13, 398-402 (2016).

35. G. Kip, A. Çelik, M. Bilge, M. Alkan, H.A. Kiraz, A. Özer, V. Şıvgın, Ö. Erdem, M. Arslan, M. Kavutçu, Dexmedetomidine protects from post-myocardial ischaemia reperfusion lung damage in diabetic rats. Libyan J. Med. 10, 27828 (2015)

36. H. Eldar-Finkelman, R. Ilouz, Challenges and opportunities with glycogen synthase kinase-3 inhibitors for insulin resistance and Type 2 diabetes treatment. Exp. Opin. Investig. Drugs 12, 1511-1519 (2003) 